\title{
INTELLIGENCE COLLECTIVE ET RECHERCHE D’INFORMATION EN CONTEXTE ORGANISATIONNEL
}

\begin{abstract}
Resumé: La recherche d'information n'est plus une activité isolée, mais s'inscrit dans un contexte plus large où recherche, dispositif, usage et partage de l'information sont pleinement liés. Également, on pense l'Intelligence Collective comme «une dynamique d'acteurs coresponsables interconnectés culturellement (soft) et organisationnellement (hard), en alliance autour de visions partagées » (Lenhardt \& Bernard, 2005, p. 31). Dans cet article, nous proposons un cas spécifique sur la question de l'intelligence collective dans une perspective de recherche d'information, en investigant quarente personnes dune très grande entreprise du secteur de l'énergie. Ils sont non-informaticiens, développeurs amateurs qui ont, en plus de leur mission principale (expertise technique, appui métier, chargé d'études, planification, etc.) une activité de production de dispositifs techniques pour répondre aux besoins locaux de leurs collègues. Les résultats de l' observation participante, dans une perspective comprehensive montre que, dans un contexte organisationnel, la structuration de la population investiguée est une réponse à un besoin informationnel hautement contextualisé. C'est aussi une manière de transformer l'organisation en favorisant une approche plus décentralisée, appuyée en cela par les dispositifs mis en place, mais surtout ancrée dans les usages et la réalité opérationnelle des acteurs (et les dynamiques associées). Nous observons une convergence entre les objectifs de l'organisation qui donnent une direction et fédèrent les acteurs en donnant un sens à leurs actions y la dimension structurelle de l'organisation qui impose des processus, des moyens, sa culture et enfin les membres eux-mêmes qui font vivre l'organisation, sa culture, qui sont les forces vives permettant d'atteindre lesdits objectifs.
\end{abstract}

Mots clés: intelligence collective; recherché d' information; context organisationnel.

\section{COLLECTIVE INTELLIGENCE AND INFORMATION RESEARCH IN ORGANIZATIONAL CONTEXT}

\begin{abstract}
The search for information is no longer an isolated activity, but is part of a larger context where research, arrangement, use and sharing of information are fully linked. In addition, we think of Collective Intelligence as "a dynamic of socially responsible actors interconnected culturally (soft) and organizationally (hard), in alliance around shared visions" (Lenhardt \& Bernard, 2005, p. 31). In this article, we offer a specific case on collective intelligence from an information research perspective, by investigating forty people from a very large company in the energy sector. They are non-expert informants, amateur developers who have, in addition to their main mission (technical expertise, business support, studies manager, planning, etc.) an activity of producing technical devices to meet the local needs of their colleagues. The results of the participant observation, from a comprehensive perspective, show that, in an organizational context, the structuring of the population investigated is a response to a highly contextualized information need. It is also a way of transforming the organization by promoting a more decentralized approach, supported in this by the mechanisms put in place, but above all anchored in the uses and the operational reality of the players (and the associated dynamics). We observe a convergence between the objectives of the organization that give direction and unite the actors by giving meaning to their actions by the structural dimension of the organization that imposes processes, means, its culture and finally the members themselves. The same people who keep the organization and its culture alive, which are the driving forces behind the achievement of these objectives.
\end{abstract}

Keywords: collective intelligence; seeking information; organizational context. 


\section{INTRODUCTION}

En 2009, le colloque organisé par le chapitre français de l'International Society of Knowledge Organization (ISKO) ${ }^{1}$ à l'ENSSIB s'intéresse à la thématique « intelligence collective et organisation des connaissances $»^{2}$ dont une session est dédiée aux questions relatives à la « recherche collaborative, veille informationnelle et partage des connaissances ». Cette rencontre scientifique a été l'occasion d'évoquer les apports spécifiques de l'intelligence collective en particulier dans des contextes organisationnels où l'information et la connaissance sont devenues des actifs immatériels. Ce cadre de l'intelligence collective interroge en effet sur la capacité des acteurs à agir collectivement au sein d'une organisation, mais aussi sur les apports de cette approche décentralisée dans le traitement de l'information et de la connaissance.

Suite à ce point d'étape, les transformations associées au déploiement des technologies de l'information et de la communication (TIC) et la médiation des activités anthropocentrées par des dispositifs techniques se sont accélérées. Fin 2019 - début 2020 marque un point de bascule avec la pandémie de Covid-19 et son rôle d'accélérateur de la transformation numérique des organisations. Celles-ci ont dû mettre en place ou renforcer leurs infrastructures numériques de sorte à assurer une continuité dans leurs activités.

Dans un premier article de 2019 (Henry, 2019b), nous avons analysé l'émergence d'une intelligence collective au sein d'une grande organisation du secteur de l'énergie, en complément dans un second article (Henry, 2019a) où nous avons analysé l'hybridation numérique de cette organisation pour étudier le contexte de faisabilité sociotechnique favorisant cette situation. En filigrane s'est tracé les contours d'un concept, 1' «expérience organisation » qui se veut l'expression d'une réflexion au niveau organisationnel quant à la capacité de ses membres à la transformer (Henry, 2021). L'angle privilégié dans cet article s'inscrira plus particulièrement sur la thématique de la recherche d'informations dans un contexte d'intelligence collective au sein d'une organisation.

La problématique de la recherche d'information est principalement traitée par deux angles (Lin, 2017) :

- L'angle technique (system-centered), qui recouvre à la fois les outils liés à la recherche d'information (avec aujourd'hui la présence croissante de l'intelligence artificielle pour

\footnotetext{
${ }^{1}$ https://www.isko-france.asso.fr/

2 http://isko-france.asso.fr/pdf/isko2009/index.html
} 
rechercher l'information (Guarino, Samet, \& Cavallucci, 2021)), à l'évaluation de ces systèmes, mais aussi aux méthodes à l'image des opérateurs booléens. L'accent est souvent mis ici sur la question de la performance de la recherche d'information. Une limite inhérente à cette approche technocentrée est la présupposition selon laquelle l'information qui est recherchée est formalisée, disponible sur un espace en ligne et que l'individu qui cherche l'information sache ce qu'il recherche, aspect indissociable du paradigme dominant dans la Recherche d'Information (RI) actuellement selon Hjørland (2021). En effet, le principe dominant serait celui du «query transformation», qui impose de connaître en amont les symboles (par exemple les mots) au sein d'un document qu'une personne souhaite trouver.

- L'angle centré utilisateur (user-centered) où l'accent est mis sur les comportements de la personne qui a un besoin informationnel, mais aussi sur des aspects cognitifs, contextuels ou personnels qui influencent sur ce processus.

Nous souhaitons déplacer ces perspectives de sorte à appréhender la RI au travers des mécanismes sociotechniques qui interviennent dans ce processus, notamment ceux qui se mettent en place au croisement entre individus (intrinsèquement pluriels) qui composent cette intelligence dite collective et les espaces, ici principalement numériques, dans lesquels ils évoluent. Ainsi, la recherche d'information n'est plus une activité isolée, mais s'inscrit dans un contexte plus large où recherche, dispositif, usage et partage de l'information sont pleinement liés.

En effet, en pensant l'Intelligence Collective comme « une dynamique d'acteurs coresponsables interconnectés culturellement (soft) et organisationnellement (hard), en alliance autour de visions partagées » (Lenhardt \& Bernard, 2005, p. 31), nous observons une convergence au sein des organisations entre : les objectifs de l'organisation qui donnent une direction et fédèrent les acteurs en donnant un sens à leurs actions ; la dimension structurelle de l'organisation ${ }^{3}$ qui impose des processus, des moyens, sa culture et enfin les membres eux-mêmes qui font vivre l'organisation, sa culture, qui sont les forces vives permettant d'atteindre lesdits objectifs.

Ainsi la problématique traitée par cet article sera liée à la recherche d'information au sein d'une organisation pour trouver de l'information fortement contextualisée qui est non formalisée

\footnotetext{
3 L’organisation est ici acceptée comme étant un « ensemble de dispositifs sociaux, juridiques, techniques, culturels, économiques, éthiques destinés à permettre à des actions socialement organisées de se déployer dans le temps et dans l'espace, pour atteindre des résultats » (Lorino, 2005, p. 55).
} 
ou non documentée. Nous mobiliserons pour ce travail une étude de cas comprenant à la fois une observation participante, et des entretiens avec des acteurs de l'organisation.

\section{CADRAGE THÉORIQUE}

\subsection{LA RECHERCHE D'INFORMATION EN CONTEXTE ORGANISATIONNEL}

Un individu met en œuvre une recherche d'information quand il/elle ressent un besoin informationnel. C'est d'autant plus prégnant dans notre contexte de " société de l'information » où l'information est un actif économique essentiel pour être compétitif (Miège, 2002). Ainsi, les travaux sur la recherche d'information (information retrieval), qui est un concept relativement ancien, se sont fortement multipliés ces dernières années, en particulier avec la massification du web (à partir des années 1990-2000) et l'accès facilité qu'il permet aux ressources en ligne.

Nous retrouvons plusieurs perspectives concernant la recherche d'information. Les travaux qui privilégient un angle informatique comme Chiaramella \& Mulhem (2007, p. 12) définissent la recherche d'information comme étant un champ de recherche qui a : «pour thème central l'étude de modèles et systèmes d'interaction entre des utilisateurs humains et des corpus de documents numériques, en vue de la satisfaction de leurs besoins d'information ». L'accent est ainsi mis sur la dimension technique marquée par le contexte informatique. Si cet angle est fortement porté par les acteurs du monde informatique avec le développement d'algorithmes et de programmes informatiques toujours plus performants pour traiter la masse d'information sans cesse croissante, il nous semble, en phase avec Lévy (1997), que l'angle de l'intelligence collective favorise un traitement complémentaire de l'information qui est plus axé sur le collectif et la résolution collective d'un problème informationnel. En effet, comme le notent Doria \& Zacklad (2010), la recherche d'information mobilise de nombreux concepts et savoirs info-documentaires qui sont mis en œuvre de manière, le plus souvent, inconsciente par les acteurs. La dimension technique seule n'est donc pas suffisante. L'écueil étant aussi de s'inscrire dans un solutionnisme technologique, en considérant qu'un dispositif qui est opérant et efficace dans un contexte l'est nécessairement dans un autre.

Pour compenser ce déficit, des approches comme la recherche collaborative d'information (Tricot \& Comtat, 2012) se sont aussi développées ces dernières années. C'est une réponse potentielle à la critique, certes ancienne, mais toujours d'actualité, de Marchionini (1992) pour qui 
les systèmes de recherche d'informations sont principalement perfectionnés pour la formulation de requêtes et l'analyse des résultats, mais ignorent pour la plupart la problématique de l'identification du manque informationnel (ou sa clarification) et l'extraction d'informations associées (même si Google se rapproche de plus en plus en fournissant non plus seulement une liste d'URL, mais dans certains cas directement la réponse à une recherche).

C'est aussi une réponse au « paradigme de l'individu », évoqué par Ben Abdallah (2012), qui met en lumière que les travaux liés à la recherche d'information se concentrent principalement sur un individu unique, sans interaction avec d'autres individus. Cette remise en cause de la pensée individualisante de la RI est aussi intéressante, eu égard de l'environnement que nous traitons, au sein des organisations. Ainsi, comme le mettait déjà en lumière Orlikowski (1996a, 1996b), c'est avant tout la culture de l'entreprise qui incite ou non au partage. C'est un facteur qui nous semble essentiel à prendre en compte dans ce contexte de recherche d'information dans le cadre d'une intelligence collective.

\subsection{DU COLLABORATIF VERS UNE INTELLIGENCE COLLECTIVE INSTRUMENTÉE}

Dans la perspective de Tricot et Comtat, la recherche d'information repose sur le principe du dialogue entre un individu (avec un besoin informationnel) et un document et/ou le système documentaire (Tricot \& Comtat, 2012). D'autres acteurs sont à même d'aider la personne présentant un besoin informationnel par le dialogue (en orientant ou posant des questions par exemple) ou en co-construisant la solution au besoin exprimé. Les auteurs soulignent ainsi la difficulté pour comprendre les mécanismes et processus cognitifs, métacognitifs, affectifs, sociaux mobilisés dans le cadre de la recherche d'information.

Le cadre de l'organisation nous permet d'insérer ici un facteur essentiel : l'orientation vers un but commun des acteurs. Comme le soulignent Maleszka \& Nguyen (2015, p. 332), « les membres du collectif sont autonomes et intelligents. Leurs connaissances peuvent être incohérentes. Les membres du collectif sont généralement orientés vers des tâches spécifiques », il est envisageable de considérer que l'organisation se prête tout particulièrement à sa formation du fait de sa capacité à générer des tâches et des buts collectifs (Woolley, Aggarwal, \& Malone, 2014). 
En effet, le contexte précédemment cité n'étant pas contextualisé, nous inscrire dans cet environnement organisationnel nous permet d'évoquer le concept d'intelligence collective plutôt que collaboratif, en l'acceptant de la manière suivante : « une intelligence partout distribuée [...], une intelligence sans cesse valorisée [...], la coordination en temps réel des intelligences [...], [pour] aboutir à une mobilisation effective des compétences » (Lévy 1997, p. 29-30). La mise en réseau des organisations en lien avec le déploiement des Technologies de l'Information et de la Communication (TIC) offre un cadre effectif à cette intelligence distribuée qui est coordonnée et qui doit aboutir à un résultat.

C'est dans cette logique que Kerckhove a construit une approche originale de l'Intelligence Collective, qu'il qualifie d'intelligence connectée qui est « la tendance d'entités séparées et antérieurement non reliées à se joindre par un lien ou une relation» (Kerckhove, 2000, p. 196). Ses travaux s'inscrivent alors dans la démocratisation de l'informatique en réseau et sont fortement marqués par les apports de Marshall Macluhan (McLuhan, 1962; McLuhan \& Powers, 1989). En acceptant Internet comme « l'art des connexions. Intel-Ligere veut dire « lier entre » c'est-à-dire faire des liens, établir des rapports, trouver des relations entre des objets ou des idées » (Kerckhove, op. cit., p. 261), il l'analyse comme « un immense système de pensée connectique dans lequel chaque individu entre à sa manière, pour ses propres fins, et ajoute au patrimoine commun ses propres marques » (ibid.).

L'intelligence collective devient alors à la fois consubstantielle à l'organisation et dans le même temps participe à sa construction. Cette inscription de l'intelligence collective dans l'organisation se traduit en particulier sous la forme des communautés, comme les communautés de pratique qui «sont par excellence l'espace de la rationalité procédurale et de la transmission. Les membres viennent y consolider des pratiques et leurs identités y sont inséparables de la maitrise de celles-ci » (Zacklad, 2016, p. 6). Elles servent aux membres de l'organisation à construire en commun du sens. Ainsi, c'est «le cœur de l'intelligence collective qui vise à rendre chaque acteur porteur de la vision, comme une cellule vivante est porteuse du code génétique » (Lenhardt \& Bernard, 2005, p. 12).

L'organisation réticulaire, c'est-à-dire en réseau, repose alors à la fois sur un environnement technique et social qui interagit, en particulier dans le cadre de la recherche d'information. Si les premiers écrits relatifs à l'intelligence collective sont marqués par une dimension religieuse (comme la Noosphère du jésuite Pierre Theilhard de Chardin (1995)), le 
développement de l'informatique en réseau favorise la pensée d'une intelligence collective instrumentée à l'œuvre.

Hosseini et al. (2015) mais aussi Curry, Freitas et O'Riain (2015) mettent en avant une déclinaison de l'intelligence collective connue sous le nom de « sagesse des foules » (Surowiecki, 2004) pour le cadre professionnel. Ainsi, ils font le parallèle avec les communautés de pratique et les communautés virtuelles dans une perspective de Knowledge Management. Pour eux, la « sagesse des foules » semble être un moyen de favoriser « les approches centralisées de l'acquisition de connaissances et de la prise de décision, notamment avec la présence de la nouvelle technologie des réseaux sociaux et de la communication de masse »(Hosseini et al., 2015, p. 122), mais aussi de prendre des décisions, résoudre des problèmes (Lopez, Belaud, Lann, \& Negny, 2015) ou obtenir du feedback critique selon les témoignages qu'ils ont recueillis.

Aujourd'hui l'Intelligence Collective est considérée comme un atout concurrentiel pour les organisations si elle est exploitée correctement (Lykourentzou, Papadaki, Vergados, Polemi, \& Loumos, 2010, p. 36). Au-delà de l'aspect mercantile, c'est aussi une approche qui rend possible une organisation décentralisée ; ce qui se traduit concrètement pour les membres de l'organisation « en faisant partie d'une communauté, les participants sont en mesure de partager les coûts, les risques et les défis techniques, tout en bénéficiant de la sagesse de la communauté et de l'effet de réseau. » (Curry, Freitas, \& O'Riain, 2015, p. 30). En portant cette structuration originale qui se détache du fonctionnement hiérarchique plus courant, c'est la possibilité pour l'organisation d'avoir une meilleure adaptation à son environnement en renforçant particulièrement l'action collective.

Lévy discerne précocement cette question en proposant comme solution de favoriser « la socialisation des résolutions des problèmes plutôt que leur traitement par des instances séparées » (Lévy, 1997, p. 68-69). Un exemple actuel est celui du traitement de la donnée, ainsi, Jung (2017) associe intelligence collective et traitement des données massives pour la collecte, l'analyse et l'utilisation de ces données. Mais ce traitement collaboratif nécessite des « outils de filtrage intelligent des données, de navigation dans l'information, de simulation de systèmes complexes, de communication transversale et de repérage mutuel des personnes et des groupes en fonction de leurs activités et de leurs savoirs » (ibid.). 


\section{MÉTHODE ET PRÉSENTATION DU CAS D'ÉTUDE}

Pour ce travail de recherche, nous nous inscrivons dans une approche compréhensive d'un cas d'étude et dont les données qualitatives ont été collectées dans le cadre d'une observation participante (Bastien, 2007 ; Lapassade, 2006) d'une durée de trois ans (2015-2018) durant laquelle des entretiens, des enquêtes et une analyse documentaire ont été réalisés. Pour cet article, nous mobiliserons plus particulièrement un travail d'enquête et des ateliers de co-design réalisés auprès des membres d'un collectif étudié pendant ces trois années :

Tableau 1 : synthèse de notre démarche méthodologique

\begin{tabular}{|c|c|}
\hline Population investiguée & $\begin{array}{l}\text { Membres d'un collectif de développeurs dits « en région } \\
\text { répartis sur l'ensemble du territoire français. C'est ur } \\
\text { population de } 40 \text { personnes environ. Non-informaticiens, ce } \\
\text { développeurs amateurs ont, en plus de leur missid } \\
\text { principale (expertise technique, appui métier, char } \\
\text { d'études, planification, etc.) une activité de production } \\
\text { dispositifs techniques pour répondre aux besoins locaux } \\
\text { leurs collègues. }\end{array}$ \\
\hline Objet de l'investigation & $\begin{array}{l}\text { Analyse de leur perception de ce collectif, en particulier, es } \\
\text { ce une intelligence collective? Analyse des besoin } \\
\text { informationnels associés. }\end{array}$ \\
\hline Méthode de l'investigation & $\begin{array}{l}\text { Observation participante dans une perspecti } \\
\text { compréhensive, ayant pour objectif de comprendre } \\
\text { profondeur le fonctionnement d'un processus. }\end{array}$ \\
\hline Collecte des données & $\begin{array}{l}\text { Travail documentaire, enquête réalisée auprès du collect } \\
\text { avec un taux de réponse de } 20 \% \text { et résultats des ateliers } \\
\text { co-design liés au collectif. }\end{array}$ \\
\hline Terrain de l'étude & Très grande entreprise du secteur de l'énergie. \\
\hline
\end{tabular}

L'organisation observée s'inscrit dans un contexte où elle se retrouve confrontée à une hybridation technique (Battilana \& Lee, 2014; Huang, Pan, \& Liu, 2017) liée aux transformations véhiculées par les technologies, mais aussi par des obligations légales (séparation des systèmes d'information des acteurs de l'énergie en lien avec l'ouverture du marché de l'énergie en France). L'hybridation se traduit alors par un effet de croisement des usages sociaux et de la technologie 
(au sens premier de l'hybridation). Dans le même temps, une cohabitation s'instaure, en effet, l'entreprise doit intégrer des logiques de fonctionnement différentes (Pache \& Santos, 2013) comme la décentralisation ou la dualité entre l'activité historique et le développement de nouveaux services. La Direction des Systèmes d'Information n'ayant pas la capacité de répondre à toutes les demandes des acteurs régionaux, ceux-ci ont mis en place un système de «shadow IT ${ }^{4}$ (Silic, Barlow, \& Back, 2016), pour développer des dispositifs sociotechniques à partir de leurs connaissances en programmation (HTML/CSS, PHP pour le web ou VBA) et de leurs connaissances « métiers » répondant à leurs besoins.

Orientée vers un même objectif, une communauté nativement virtuelle (Rheingold, 1995) est créée en 2015 sur le réseau social interne de l'organisation. Instanciée par deux développeurs (l'un à Lyon, l'autre à Paris), nous retrouvons cette mise en réseau des intelligences évoquées par Kerckhove, ainsi, elle aurait difficilement pu voir le jour sans les TIC et la baisse des coûts de coordination ou de participation à l'action collective induits par leur utilisation (Lupia \& Sin, 2003).

La diversité du groupe est assurée par l'hétérogénéité des profils des membres répartis dans plusieurs régions, ce qui autorise d'une part à une multiplicité de besoins de s'exprimer et d'autre part de proposer un éventail de solutions opérationnelles. Les membres du collectif implémentent des dispositifs en réponse aux besoins de leurs collègues en région, leur force est de pouvoir proposer ainsi des solutions à des besoins non formalisés, mais qui facilitent le travail dans les équipes. Grâce à la communauté, les développeurs partagent leurs réalisations, leurs problématiques et s'informent.

La participation à la communauté en ligne ou aux évènements en présentiel est ouverte à tous. Ce sont des espaces d'échanges et de rencontres d'autant plus importants que la plupart développent seuls dans leur service. Pour présenter et partager les différents projets sur lesquels ils ont pu travailler, un portail a été mis en ligne sur lequel les applications qu'ils développent sont présentées. Si d'autres services sont intéressés, il est alors possible de partager le code source pour qu'ils puissent l'utiliser. Les décisions se prennent de gré à gré et reposent sur un contrat tacite de réciprocité comme indiqué dans le manifeste du groupe. 4 Le «shadow IT » recouvre l'ensemble du matériel informatique, logiciels utilisé par des salaries de
l'organisation et qui n'est pas approuvé par le service informatique. 


\section{UNE RECHERCHE D'INFORMATION DÉCENTRALISÉE ET CONTEXTUALISÉE}

\subsection{UNE INFORMATION CONTEXTUALISÉE POUR RÉPONDRE AU BESOIN DU COLLEFIT}

Concernant le cadre précis de ce collectif, dans l'enquête réalisée, 20\% de répondants sur la globalité du collectif, l'ensemble des participants considère explicitement que ce collectif est une intelligence collective. Il est intéressant de noter que dans les justifications relatives à cette affirmation, trois développeurs mettent spécifiquement en avant que :

- « Réfléchir à plusieurs à la même problématique permet de faire ressortir des solutions auxquelles nous n'aurions pas pensé. »

- «L'entraide mutuelle qui permet d'avancer sur son travail et d'aider un collègue qui rencontre le même problème. »

- «Mettre les connaissances de tous au service de tous. »

Les membres du collectif mettent spontanément en avant la résolution collective de problèmes (évoquée par Lévy en 97), la mise en commun de la connaissance et un accès à une information contextualisée. En effet, bénéficier d'une information contextualisée et pertinente pour leurs besoins est un facteur essentiel pour les participants. L'ensemble des répondants citent explicitement qu'ils cherchent, au travers du collectif, de l'information et à la partager. Nous pouvons aussi souligner l'importance de l'objectif commun (mais aussi des accointances personnelles), en effet, sur les répondants, ils sont nombreux à mettre en lumière qu'ils ont rejoint le collectif :

- Par intérêt personnel : goût/passion pour les technologies et le numérique ;

- Par intérêt professionnel : connaissance des autres développeurs, suggestion par un responsable.

Ces deux types de facteurs ne sont pas antagonistes, ils sont plusieurs à mentionner aussi bien un intérêt personnel que professionnel. Ce point peut illustrer aussi la tension croissante entre vie privée/vie professionnelle (Le Douarin, 2007) même si nous ne développerons pas précisément ce point.

Dans le cadre de cette recherche d'informations basée sur une perspective d'intelligence collective, l'intérêt est autant l'information en elle-même que les acteurs qui la partagent. En effet, 
dans le cadre d'une organisation, en particulier ici sur des points techniques, les développeurs en région recherchent une information hautement contextualisée, information qu'ils ne pourraient trouver hors de leur organisation. Ainsi, sur la figure $\mathrm{n}^{\circ} 1$, nous illustrons un exemple d'information partagée dans une base de connaissances :

Figure 1 : exemple de ressources mises à disposition sur le SharePoint de la communauté

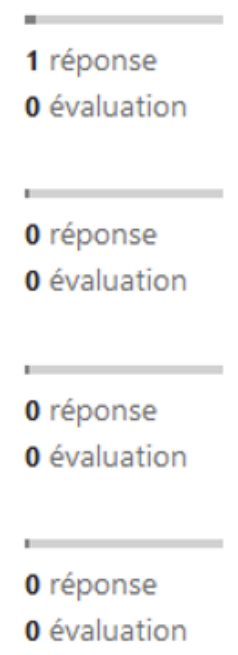

Quelques commandes utiles pour passer des paquets dans le proxy GRDF Bashexport proxy= :8080export https_proxy=http://p... Par Dernière réponse par 28 janvier

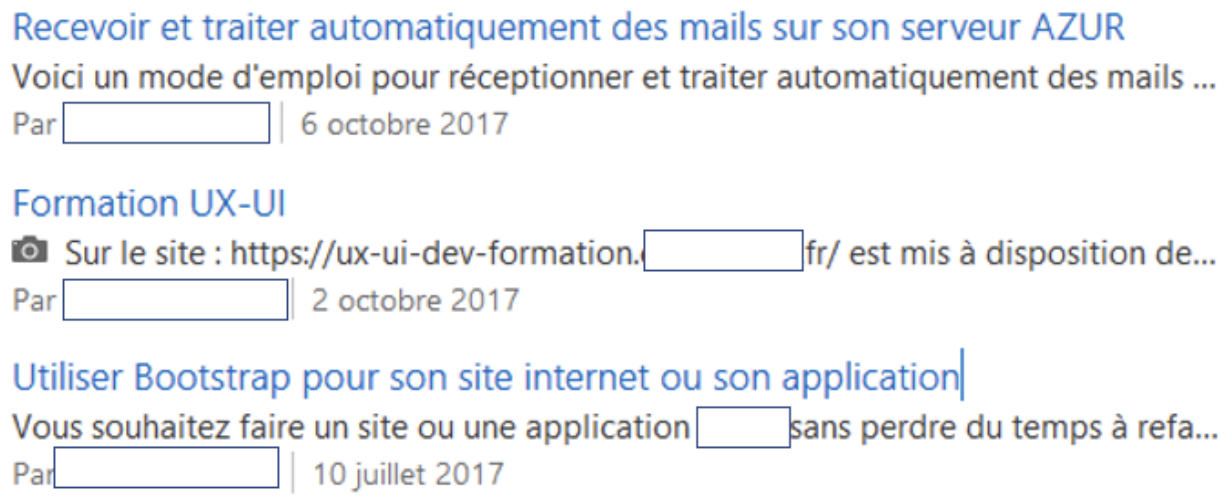

L'approche par l'intelligence collective de la recherche d'information favorise, in fine, une réponse personnalisée et contextualisée dans cette organisation. En associant celle-ci à un système d'organisation des connaissances (SOC) ou à une démarche de Knowledge Management, l'organisation tout entière peut bénéficier des réponses proposées. Dans le cadre de l'exemple présenté, c'est l'outil SharePoint qui a été mobilisé pour cette démarche de capitalisation des connaissances. Les développeurs s'inscrivent plus dans une logique d'entraide qui entraîne de facto des situations d'apprentissage par les pairs, qu'il est possible d'assimiler à l'apprentissage collaboratif, les acteurs mettant en place une coopération pour leur apprentissage (Sidir \& Papy, 2006) avec un support numérique.

Cette appropriation des dispositifs techniques nous permet d'illustrer la capacité de ces acteurs à construire leurs propres outils en fonction de leurs besoins. Si d'autres outils sont proposés par l'organisation (comme l'intranet ou des wikis), ils ne répondent pas forcément aux besoins informationnels exprimés par les acteurs. Ils ont alors détourné le réseau social, Yammer, et se sont appuyés sur le système de gestion électronique de documents (SharePoint) associé pour construire un environnement informationnel favorisant la recherche et le partage d'information. 
Dans le cas de ce collectif, nous retrouvons bien l'idée selon laquelle « la compétence collective des acteurs est basée sur l'existence de réseaux qui assurent le partage des connaissances » (Alter, 2000, p. 267). Les membres de cette intelligence collective sont confrontés à des besoins informationnels pour mener à bien leurs tâches et leurs missions. Ils mettent en œuvre un système à la fois social et technique pour obtenir l'information dont ils ont besoin. Pour cela, ils se sont constitués en collectif et ont construit des dispositifs liés à la connaissance en lien avec leurs usages (approche « dans l'usage » pour Folcher (2015)). Cette approche est alors une source de légitimation pour le système et un moyen de partager le point de vue de la communauté sur la façon dont elle organise les connaissances avec le reste de l'organisation.

\subsection{STRUCTURATION EN RÉSEAU}

Dans le cas évoqué, la décentralisation de la recherche d'information accompagne la décentralisation de l'organisation. En effet, la réticularisation de l'entreprise met en œuvre un double processus :

- Technique avec la mise en place des infrastructures numériques inhérentes à ce fonctionnement. Ainsi, ces outils techniques « ont élargi les potentialités puisqu'il est aujourd'hui possible de travailler ensemble, à une très grande échelle, sur un même projet, mais à distance, de manière synchrone ou asynchrone, sur des supports modifiables tout en permettant une relation directe entre les " collaborateurs " ou " coopérants " grâce à Internet »(Gangloff-Ziegler, 2009, p. 95).

- Social avec l'apprentissage et l'appropriation (voir le détournement ou catachrèse) desdits dispositifs techniques. Ainsi, le collectif étudié s'est approprié et même à détourner les objectifs initiaux des outils mis en œuvre. Yammer (le réseau social numérique utilisé) est devenu un espace de recherche et d'acquisition d'informations.

L'information prend alors une valeur collaborative : dans ce cas, la valeur de l'information provient de sa diffusion et de la capacité des personnes à l'intégrer et à l'utiliser dans le cadre de leurs activités. Cette approche favorise ainsi une organisation décentralisée de la recherche d'information où la recherche d'information se fait pratiquement dans une perspective « glocale ». Elle est favorisée en cela par les TIC et leur effet décentralisateur (Huang et al., 2017; Prasarnphanich \& Gillenson, 2003). Le rôle du dispositif est alors d'accompagner l'intelligence 
collective en émergence ; c'est une réponse effective à l'évolution de l'organisation et à sa réalité opérationnelle (sur la majeure partie du territoire français). À travers cette intelligence collective émergente, l'organisation étudiée accentue sa réticulation et tende alors à se rapprocher d'une entité en réseau, voire d'une méta-organisation constituée de collectifs intelligents orientés vers l'atteinte de ses objectifs. La synthèse entre l'instrumentation numérique à vocation sociotechnique et l'intelligence collective se révèle être une expression futurible de l'organisation en voie de transformation vers une méta-organisation.

La médiation instrumentale s'inscrit dans un contexte à la fois historique (histoire et choix de l'organisation), technique (notamment les conditions préalables identifiées par Akrich (1991)) et culturel (culture de l'entreprise, contexte du secteur d'activité), au point qu'il est concevable de considérer que « l'apparition des nouvelles pratiques se greffe sur le passé, sur des routines, sur des survivances culturelles qui perdurent et continuent à se transmettre bien au-delà de leur apparition » (Mallein \& Toussaint, 1994, p. 317).

En travaillant sur les dispositifs, et en particulier les systèmes d'information, nous en arrivons à considérer que pour concevoir un système pertinent pour l'utilisateur final, il convient « de mobiliser le principe téléologique et donc de s'interroger sur les finalités du projet de connaissance, qui doit être en adéquation avec la construction collective du sens de sorte à adapter le système aux problématiques rencontrées par les acteurs »(Verlaet, 2015, p. 253). Ce principe téléologique est d'ailleurs au centre de la philosophie de la théorie de l'action (Habermas, 1984) que Wertsch (1991) réexploite dans ses travaux sur l'action médiée, avec une perspective socioculturelle. L'enjeu étant la décision entre les différentes possibilités d'action qui existent pour l'atteinte d'un objectif identifié au préalable. Corrélé à ce principe téléologique et se basant sur la métaphore dramaturgique (dramaturgical metaphor) de Goffman (1959), Habermas (1984) développe le concept d'action dramaturgique (dramaturgical action) où l'acteur souhaite véhiculer une image, une impression afin de provoquer des évocations subjectives parmi les témoins de l'action. Une autre forme d'action existe pour Habermas : les actions régulées par la norme (normatively regulated action) qui sont alors des actions non pas individuelles, mais celles d'un groupe social qui les orientent vers des valeurs communes. Pour être partie prenante du groupe, l'individu doit se soumettre aux normes et adopter un comportement compatible.

Ces trois formes d'actions sociales (téléologique, normatif, dramaturgique) et la quatrième, la forme communicationnelle, se manifestent au sein des dispositifs sociotechniques qui reposent sur 
ledit principe téléologique et contribuent à véhiculer une image de l'utilisateur en lui donnant une capacité à agir tout en le contrôlant par des normes présentes de facto.

Ce dernier point nous amène à mobiliser la question éthique, en particulier ici une éthique avec une forme d'engagement de réciprocité par les membres de cette intelligence collective. En effet, au sein du groupe se met en place une forme de régulation par les pairs qui repose sur un contrat tacite où chaque membre s'engage à contribuer au collectif à la hauteur de ses possibilités. Une tolérance existe toutefois, en effet, les contributeurs étant bénévoles (ils n'ont pas de valorisation autre que la valorisation sociale et un accès privilégié à une information utile pour leurs missions). C'est un réseau qui repose sur une structuration faible où les personnes peuvent entrer/sortir simplement voire faire des contributions très ponctuelles. En effet, sur Yammer, eu égard au fonctionnement en tant que communauté ouverte, de nombreuses personnes sont membres alors même qu'elles ne contribuent pas à cette intelligence collective. Seul un nombre restreint de développeurs (environ 40) participent activement en dehors des questions techniques posées sur le fil de discussion. Les échanges par messages électroniques, les rencontres physiques où ne sont présents que leurs pairs génèrent un volume plus important d'interactions, d'échanges et d'apprentissage, similaire à la situation identifiée dans le Vill@ge par Boboc, Gire, Rosanvallon, \& Rosanvallon (2015) et illustré par d'importants pics de consultation correspondent aux différentes journées où les membres se rencontrent.

Plusieurs dynamiques existent en lien avec cette intelligence collective :

Une dynamique interne où les protagonistes recherchent, échangent de l'information et produisent des connaissances liées au développement informatique dans l'environnement technique de l'organisation (i.e : connexion au proxy de l'entreprise, utilisation du système d'authentification, paramétrage des serveurs, etc.) nécessaires à la bonne réalisation de leurs missions, mais aussi se mettent en réseau dans le dessein de favoriser la socialisation et la production collective. Dans l'enquête, les entretiens et les ateliers, plusieurs tendances transparaissent : leurs envies avec une volonté de partage, de co-construction, d'entraide dans leur quotidien, d'informations contextualisées à leur environnement et leurs attentes comme les afterworks, les rencontres, les formations qui sont pour eux autant d'occasions de se coudoyer et de renforcer leurs liens. Une dimension « outil » est aussi présente (code source, forum, tutoriaux, Massive Open Online Course) qui traduit la dimension technique de la communauté. Des outils technologiques (SharePoint ou Yammer) ont permis la formation de la communauté : modalités de partage, de travail et de formation. Chaque 
membre est libre de créer une page sur le SharePoint de façon à expliciter la connaissance qu'il souhaite partager pour la transformer, par l'action collective en connaissance organisationnelle utilisable par tout un chacun. Lors de la réalisation d'une page, ils s'inscrivent dans une activité constructive en constituant des ressources utiles pour leurs futures actions productives.

- Une dynamique « métier ». Étant donné leur présence au sein des entités régionales, les développeurs sont au plus près de la réalité opérationnelle, qu'ils comprennent et dont ils partagent le système de représentation. Les réalisations qu'ils font, qui sont assimilables à du shadow IT pour la Direction des Services Informatiques (DSI), sont le plus souvent appréciées par leurs utilisateurs qui souhaitent faire intégrer au patrimoine applicatif national le dispositif sociotechnique réalisé localement. Cette dynamique est un moteur de la participation des membres à cette intelligence collective et aux besoins informationnels qu'ils rencontrent.

- Une dynamique externe avec les autres acteurs de l'organisation et particulièrement la DSI. En s'ouvrant vers la communauté en 2017, la DSI - et donc par extension en acceptant son existence, sa raison d'être - a réalisé une première étape pour légitimer l'activité des développeurs en région et cela sans absorber ou faire disparaitre cette intelligence collective. Ainsi, la DSI depuis janvier 2017 se positionne en facilitatrice et contribue à la vie de ce collectif en mettant à disposition des ressources et des infrastructures (i.e. : immersions à la DSI ; fourniture de dispositifs facilitant le développement). Dans le même temps, la vie de la communauté est renforcée par de nouvelles journées consacrées aux développeurs en région auxquelles la DSI apporte un soutien logistique et financier. Ces journées nationales à Paris $(2015,2016$, 2017) se sont vues déclinées en 2017 et 2018 plus localement pour toucher des salariés n'ayant jamais fait le déplacement jusqu'à Paris. Cette dynamique s'inscrit dans le sens du renforcement des liens entre la communauté et la DSI. C'est une manière de légitimer leurs contributions à l'organisation, de les accompagner, mais aussi d'avoir une meilleure vision des besoins des utilisateurs. Cette dynamique externe met aussi en exergue les tensions qui existent entre la vision et les arbitrages pris au siège de l'organisation et les besoins des acteurs confrontés à la réalité opérationnelle. Dans les ateliers de co-design, ces tensions ont émergé à plusieurs reprises avec une demande d'un engagement plus important de la DSI pour soutenir les projets de la communauté. 


\section{DISCUSSION DE NOS RÉSULTATS}

Ce travail de recherche sur une intelligence collective au sein d'une grande organisation met l'accent sur les apports de cette perspective par rapport à des besoins informationnels très pointus, car fortement contextualisés à l'organisation et à son fonctionnement. Il entend apporter des éléments d'analyse et de réponse à la fois sur des aspects sociaux et techniques et l'articulation entre les deux.

Si les membres du collectif notent l'intérêt pour eux de rechercher de l'information en contexte d'intelligence collective avec des réponses contextualisées, voire co-construites, en fonction des situations, il est nécessaire de nuancer certains points, notamment en lien avec les freins qui peuvent exister. Une première nuance à apporter est l'importance, même si nous pouvons considérer qu'elle est une intelligence collective instrumentée, donnée au présentiel, à la rencontre lors d'évènements organisés pour que les membres se côtoient. C'est même un élément qui peut renforcer l'envie de contribuer au collectif comme le note un développeur interrogé. Nous retrouvons ici la question de la confiance évoquée par Wenger, McDermott, \& Snyder (2002), mais aussi Larsen \& McInerney( 2002) qui mettent en lumière la difficulté pour un collectif qui se connait uniquement de manière virtuelle de développer une confiance en son sein. Cette importance se remarque aussi par la hausse des messages sur l'espace en ligne en lien avec la tenue des évènements en physique comme l'illustre la figure $\mathrm{n}^{\circ} 2$ : 
PontodeAcesso: Revista do Instituto de Ciência da Informação da UFBA

DOI: https://doi.org/10.9771/rpa.v15i3.47476

Figure 2 : Volume des messages publiés sur le groupe Yammer de la communauté Entraide des développeurs entre début 2016 et fin 2017

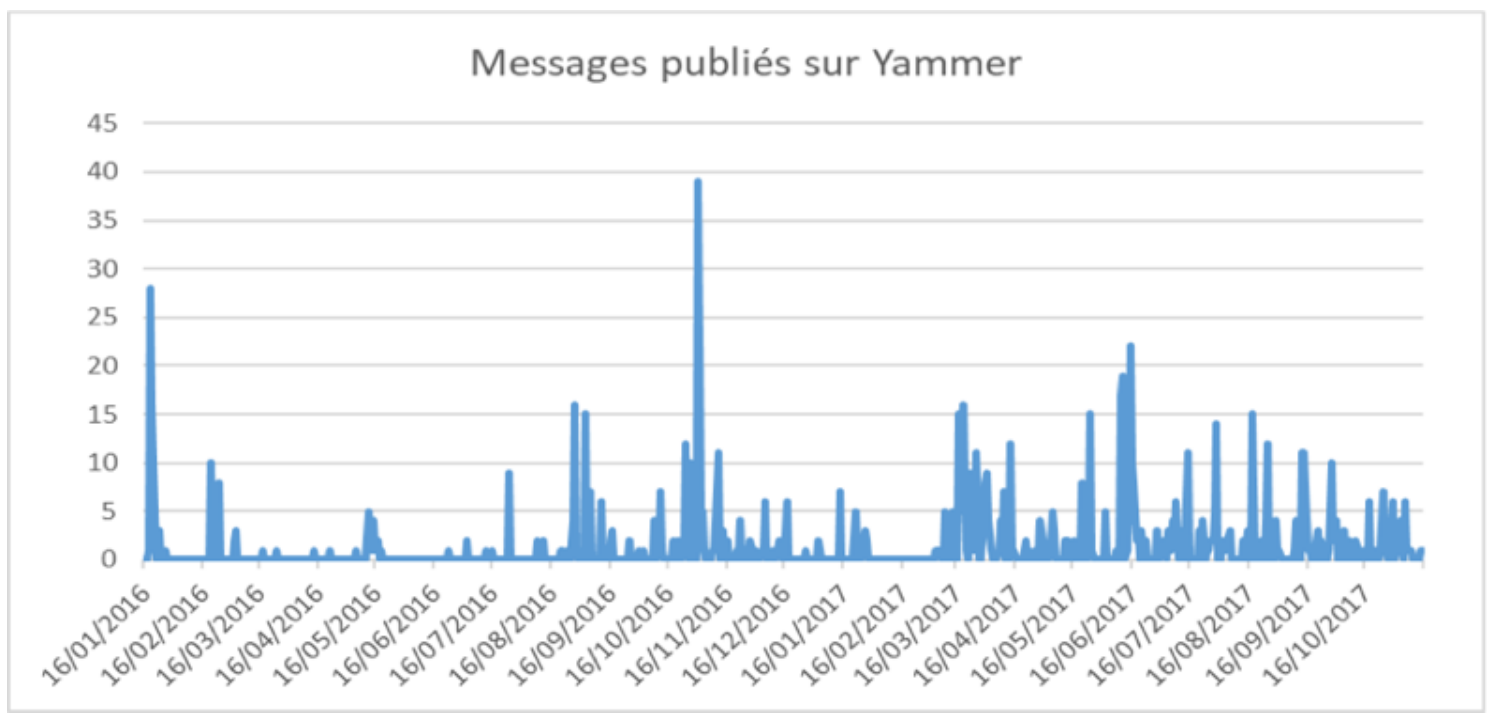

Même si la communauté est nativement virtuelle, nous pouvons noter que les pics les plus importants sont associés aux évènements en présentiel de la communauté. Ces rencontres sont alors des vecteurs de l'engagement et sont fédératrices. Elles sont notamment l'occasion pour les participants de questionner des aspects fondamentaux de cette intelligence collective, en particulier l'affirmation d'un sens partagé (qui est un risque identifié s'il n'est pas commun à l'ensemble des participants) comme l'illustre la figure $n^{\circ} 3$ : 
Figure 3 : retours des participants à un atelier de co-design du 18 octobre 2016

\begin{tabular}{|c|c|c|}
\hline & SATISFACTIONS & FREINS \\
\hline \multirow[t]{2}{*}{ 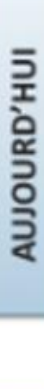 } & $\begin{array}{l}\text {-Existence d'une communauté } \\
\text { - Encouragement de la DSi envers les dev. } \\
\text {-Yammer fonctionnel et facilement accessible } \\
\text { - Réponse à un besoin commun } \\
\text { - Partage de bonnes pratiques } \\
\text { - Entraide } \\
\text {-Volontariat naissant } \\
\text { - Climat jovial et détendu } \\
\text { - Journée des développeurs déjà en place }\end{array}$ & $\begin{array}{l}\text { - Peu de communication sur la communauté } \\
\text {-Freins des managers } \\
\text {-Pas assez de rencontre physique entre les dev. } \\
\text {-Manque de temps dédié à cette activité } \\
\text {-Un seul vecteur d'informations (Yammer) } \\
\text { - Peu de volontaires } \\
\text { - Vocabulaire employé trop technique }\end{array}$ \\
\hline & ACTIONS À MENER & RISQUES IDENTIFIÉS \\
\hline$\frac{z}{\frac{z}{\Delta}}$ & $\begin{array}{l}\text {-Mettre à disposition des tutoriaux vidéos } \\
\text { - Partager les sources et les templates } \\
\text { - Dédier officiellement du temps à cette activité } \\
\text { - Reconnaitre la mission par le management } \\
\text { - Organiser des événements (afterworks, ...) et des } \\
\text { rencontres } \\
\text { - Faciliter le partage via d'autres vecteurs (ex: Twitter) } \\
\text { - Organiser des vidéos/ conférences sur un thème voté } \\
\text { (sur Yammer par ex.) } \\
\text {-Mettre en place une plateforme avec publication de } \\
\text { contenu par thème } \\
\text { - Disposer de forts appuis (sponsors) } \\
\text { - Mettre en place des formations (internes et/ou } \\
\text { externes) } \\
\text {-Avoir connaissance des projets des autres dev. } \\
\text {-Avoir connaissance des compétences de chacun }\end{array}$ & $\begin{array}{l}\text {-Manque de temps dédié à cette activité } \\
\text {-Freins organisationnels } \\
\text {-Dimensionnement SI non adapté } \\
\text { - Communauté perdue au milieu des autres (Yammer) } \\
\text { - Risque de lassitude si manque d'activité } \\
\text {-Manque de budget alloué } \\
\text {-Manque de communication autour de la } \\
\text { communauté } \\
\text {-Beaucoup d'intéressés mais peu de passionnés } \\
\text {-Manque de cohésion ou de sens (à quoi sert la } \\
\text { communauté ?) }\end{array}$ \\
\hline
\end{tabular}

Se pose aussi de manière prégnante la question du temps et de la temporalité. Cette question du temps renvoie aux fiches de poste et au temps qui est dédié effectivement par ces personnes aux travaux en commun. Plusieurs notent dans leurs réponses le manque de temps qu'ils peuvent consacrer au collectif. De manière corolaire avec le manque de temps disponible, il peut arriver que des questions ne reçoivent pas de réponses rapides. Ce qui peut être un point bloquant ou une frustration pour un développeur qui doit répondre rapidement à une problématique.

En parallèle, dans le cadre de cette intelligence collective qui s'est auto-organisée, les participants relèvent aussi des freins liés à l'encadrement et à la structure même de l'organisation (très centralisée et hiérarchisée). Nous pouvons toutefois noter que cet exemple témoigne d'une ouverture progressive de l'organisation en offrant un cadre plus perméable avec une configuration élargie d'acteurs pour la résolution d'un problème. C'est alors une première étape vers l'organisation distribuée dans une logique d'action collective. Processus à long terme, l'exemple étudié illustre alors une organisation à la fois sociale et technique à même de favoriser des « transformations en profondeur des connaissances [...] des uns et des autres du fait des apprentissages croisés permis par les dispositifs formels d'interaction » (Hubert, 2002, p. 58). Il en résulte que cette structuration, en s'appuyant sur des dispositifs sociotechniques contribuent à 
une réponse rapide aux évolutions permanentes de l'écosystème. Selon cette conception, le système interne de valeur change progressivement pour faire évoluer les routines, les règles ou les fonctionnements actuels qui lui sont inhérents et ainsi l'adapter aux besoins actuels de l'organisation.

\section{CONCLUSION}

Dans cet article, nous proposons un cas spécifique pour travailler sur la question de l'intelligence collective dans une perspective de recherche d'information. Notre proposition tend à prendre en compte à la fois les aspects sociaux (ici matérialisés par la formation d'une intelligence collective auto-organisée) et les aspects techniques (dispositifs mis en place pour l'instrumenter et l'outiller) en lien avec la sociotechnique. L'orientation vers un objectif commun et des besoins informationnels communs a favorisé l'émergence du collectif étudié, qui s'est structuré et organisé. Nous mettons alors en lumière de quelle manière, dans un contexte organisationnel, cette structuration est une réponse à un besoin informationnel hautement contextualisé. C'est aussi une manière de transformer l'organisation en favorisant une approche plus décentralisée, appuyée en cela par les dispositifs mis en place, mais surtout ancrée dans les usages et la réalité opérationnelle des acteurs (et les dynamiques associées).

\section{BIBLIOGRAPHIE}

Akrich, M. (1991). L'analyse socio-technique. In D. Vinck (Ed.), La gestion de la recherche (pp. 339-353). Bruxelles: De Boeck.

Alter, N. (2000). L’innovation ordinaire. Paris: Presses Universitaires de France.

Battilana, J., \& Lee, M. (2014). Advancing Research on Hybrid Organizing - Insights from the Study of Social Enterprises. Academy of Management Annals, 8(1), 397-441. https://doi.org/10.1080/19416520.2014.893615

Ben Abdallah, N. (2012). Réflexions sur l'analyse des pratiques informationnelles collaboratives. Les Cahiers Du Numérique, 8(1-2), 131-158. https://doi.org/10.3166/lcn.8.1$\underline{2.131-158}$

Boboc, A., Gire, F., Rosanvallon, J., \& Rosanvallon, J. (2015). Les réseaux sociaux numériques. Vers un renouveau de la communication dans les entreprises? Sociologies Pratiques, 30(1), 19-32. https://doi.org/10.3917/sopr.030.0019 
Chardin, P. T. de. (1995). Le Phénomène humain. Paris: Seuil.

Chiaramella, Y., \& Mulhem, P. (2007). La recherche d'information. De la documentation automatique à la recherche d'information en contexte. Document Numérique, 10(1), 11-38. https://doi.org/10.3166/dn.10.11-38

Curry, E., Freitas, A., \& O'Riain, S. (2015). The Role of Community-Driven Data Curation for Enterprises. In D. Wood (Ed.), Linking Enterprise Data (Vol. 1, pp. 25-47). https://doi.org/10.1017/CBO9781107415324.004

Doria, O. D., \& Zacklad, M. (2010). Améliorer la recherche d'information à l'aide de thésaurus «ad hoc». Document Numérique, 13(2), 13-40. https://doi.org/10.3166/dn.13.2.13$\underline{40}$

Folcher, V. (2015). Conception pour et dans l'usage : la maîtrise d'usage en conduite de projet. Revue Des Interactions Humaines Médiatisées, 16(1), 39-60.

Gangloff-Ziegler, C. (2009). Les freins au travail collaboratif. Marché et Organisations, 10(3), 95-112. https://doi.org/10.3917/maorg.010.0095

Goffman, E. (1959). The presentation of self in everyday life. New York: Doubleday Anchor. Guarino, G., Samet, A., \& Cavallucci, D. (2021). Patent Specialization for Deep Learning Information Retrieval Algorithms (Y. Borgianni, B. Stelian, D. Cavallucci, \& P. Livotov, Eds.). https://doi.org/10.1007/978-3-030-86614-3_13

Habermas, J. (1984). The theory of communicative action: reason and the rationalization of society. Boston: Beacon Press.

Henry, A. (2019a). Hybridation numérique d'une organisation énergétique : Analyse de son contexte de faisabilité sociotechnique. Les Cahiers Du Numérique, 15, 113-136.

https://doi.org/10.3166/LCN.15.4.113-136

Henry, A. (2019b). L'émergence d'une Intelligence Collective dans une grande entreprise de l'énergie : étude de cas d'une communauté d'entraide oeuvrant entre global et local.

Information, Organisation, Connaissances, 2(1), 1-16.

https://doi.org/10.21494/ISTE.OP.2019.0399

Henry, A. (2021). Platform and Collective Intelligence. London: ISTE Editions.

Hjørland, B. (2021). Information Retrieval and Knowledge Organization: A Perspective from the Philosophy of Science. Information, 12(3), 135. https://doi.org/10.3390/info12030135

Hosseini, M., Moore, J., Almaliki, M., Shahri, A., Phalp, K., \& Ali, R. (2015). Wisdom of the Crowd within enterprises: Practices and challenges. Computer Networks, 90, 121-132.

https://doi.org/10.1016/j.comnet.2015.07.004

Huang, J. S., Pan, S. L., \& Liu, J. (2017). Boundary permeability and online-offline hybrid 
organization: A case study of Suning, China. Information and Management, 54(3), 304-316. https://doi.org/10.1016/j.im.2016.08.002

Hubert, B. (2002). Le traitement du long terme et de la prospective (suite). Les rapports entre chercheurs et acteurs. Natures Sciences Sociétés, 10(4), 51-62.

Jung, J. J. (2017). Computational Collective Intelligence with Big Data : Challenges and Opportunities. Future Generation Computer Systems, 66, 87-88.

https://doi.org/10.1016/j.future.2016.08.021

Kerckhove, D. de. (2000). L'intelligence des Réseaux. Paris: Editions Odile Jacob.

Lapassade, G. (2006). L'observation participante. In R. Hess \& G. Weigand (Eds.),

L'observation participante dans les situations interculturelles (pp. 13-32). Paris: Economica. Larsen, K. R. T., \& McInerney, C. R. (2002). Preparing to work in the virtual organization. Information and Management, 39(6), 445-456. https://doi.org/10.1016/S0378$\underline{\text { 7206(01)00108-2 }}$

Le Douarin, L. (2007). Les chemins de l'articulation entre vie privée et vie professionnelle. Réseaux, 2007/1(140), 101-132. https://doi.org/10.3917/res.140.0101

Lenhardt, V., \& Bernard, P. (2005). L'intelligence collective en action. Paris: Pearson Education France.

Lévy, P. (1997). L'intelligence collective : Pour une anthropologie du cyberspace. Paris: La Découverte.

Lin, S. (2017). Human - Computer Interaction Research in Information Retrieval. 9-12. https://doi.org/10.1081/E-ELIS4-120044556

Lopez, R., Belaud, J., Lann, J. Le, \& Negny, S. (2015). Using the Collective Intelligence for inventive problem solving: A contribution for Open Computer Aided Innovation. Expert Systems with Applications, 42, 9340-9352. https://doi.org/10.1016/j.eswa.2015.08.024

Lorino, P. (2005). Théories des organisations, sens et action : le cheminement historique, du rationalisme à la genèse instrumentale des organisations. In P. Lorino \& R. Teulier (Eds.), Entre connaissance et organisation : l'activité collective (pp. 54-71). Paris: La Découverte.

Lupia, A., \& Sin, G. (2003). Which Public Goods are Endangered?: How Evolving Communication Technologies Affect The Logic of Collective Action. Public Choice, 117(3/4), 315-331. https://doi.org/10.1023/B:PUCH.0000003735.07840.c7

Lykourentzou, I., Papadaki, K., Vergados, D. J., Polemi, D., \& Loumos, V. (2010). CorpWiki: A self-regulating wiki to promote corporate collective intelligence through expert peer matching. Information Sciences, 180(1), 18-38. https://doi.org/10.1016/j.ins.2009.08.003

Maleszka, M., \& Nguyen, N. T. (2015). Integration computing and collective intelligence. Expert Systems with Applications, 42(1), 332-340. https://doi.org/10.1016/j.eswa.2014.07.036 
Mallein, P., \& Toussaint, Y. (1994). L'intégration sociale des technologies d'information et de la communication : une sociologie des usages. Tis, Vol. 6, pp. 316-335.

Marchionini, G. (1992). Interfaces for end-user information seeking. Journal of the American Society for Information Science, 43(2), 156-163. https://doi.org/10.1002/(SICI)10974571(199203)43:2<156::AID-ASI8>3.0.CO;2-U

McLuhan, M. (1962). The Gutenberg galaxy: the making of typographic man. Londres: Routledge.

McLuhan, M., \& Powers, B. (1989). The global village: transformation in world life and media in the 21st century. New York: Oxford University Press.

Miège, B. (2002). La société de l'information : toujours aussi inconcevable. Revue Européenne Des Sciences Sociales, XL-123, 41-54. https://doi.org/10.4000/ress.606

Orlikowski, W. J. (1996a). Improvising organizational transformation over time: a situated change perspective. Information System Research, 7(1), 63-91.

Orlikowski, W. J. (1996b). Learning From Notes: Organizational Issues in Groupware Implementation. In R. Kling (Ed.), Computerization and controversy (pp. 173-189). New York: Academic Press.

Pache, A.-C., \& Santos, F. (2013). Inside the Hybrid Organization: Selective Coupling as a Response to Competing Institutional Logics. Academy of Management Journal, 56(4), 9721001. https://doi.org/10.5465/amj.2011.0405

Prasarnphanich, P., \& Gillenson, M. L. (2003). The hybrid clicks and bricks business model. Communications of the ACM, 46(12), 178-185. https://doi.org/10.1145/953460.953498

Rheingold, H. (1995). Les Communautés virtuelles. Addison-Wesley France.

Sidir, M., \& Papy, F. (2006). De l'analyse des traces à l'analyse des usages dans un environnement éducatif médiatisé. Revue Informations, Savoirs, Décisions, Médiations, 25, $1-10$.

Silic, M., Barlow, J. B., \& Back, A. (2016). A new perspective on neutralization and deterrence: Predicting shadow IT usage. Information and Management, 54(8), 1023-1037. https://doi.org/10.1016/j.im.2017.02.007

Soulé, B. (2007). Observation participante ou participation observante ? Usages et justifications de la notion de participation observante en sciences sociales. Recherches Qualitatives, 27(1), 127-140.

Surowiecki, J. (2004). La sagesse des Foules. Paris: JC Lattès.

Tricot, A., \& Comtat, P. (2012). La recherche d'information comme dialogue. Les Cahiers Du Numérique, 8(1), 15-38. https://doi.org/10.3166/1cn.8.1-2.15-38 
Verlaet, L. (2015). La deuxième révolution des systèmes d'information : vers le constructivisme numérique. Hermès, La Revue, 71(1), 249-254.

Wenger, E., McDermott, R., \& Snyder, W. M. (2002). Cultivating communities of practice. Boston: Harvard Business School Press.

Woolley, A. W., Aggarwal, I., \& Malone, T. W. (2014). Collective Intelligence in Teams and Organizations. In T. W. Malone \& M. S. Bernstein (Eds.), The handbook of collective intelligence (pp. 143-158). Cambridge: MIT Press.

Zacklad, M. (2016). Diversité des ontologies de la communication et de l'action collective. Revue Française Des Sciences de l'information et de La Communication, 9. https://doi.org/10.4000/rfsic.2419 